\title{
Politique et sociétés à Chypre aujourd'hui : une introduction
}

\section{Mathieu Petithomme}

\section{Q OpenEdition}

1 Journals

Édition électronique

URL : https://journals.openedition.org/ceb/13593

DOI : $10.4000 /$ ceb. 13593

ISSN : 2261-4184

Éditeur

INALCO

Édition imprimée

ISBN : 9782858313341

ISSN : 0290-7402

Référence électronique

Mathieu Petithomme, «Politique et sociétés à Chypre aujourd'hui : une introduction », Cahiers

balkaniques [En ligne], 46 | 2020, mis en ligne le 25 février 2020, consulté le 06 juillet 2021. URL : http:// journals.openedition.org/ceb/13593; DOI : https://doi.org/10.4000/ceb.13593

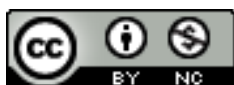

Cahiers balkaniques est mis à disposition selon les termes de la Licence Creative Commons Attribution - Pas d'Utilisation Commerciale 4.0 International. 


\title{
Politique et sociétés à Chypre aujourd'hui : une introduction
}

\author{
Mathieu Petithomme \\ Université de Franche-Comté
}

Plus de 45 ans après la partition de 1974, les enjeux qui taraudent les sociétés chypriotes grecque et turque demeurent nombreux: division et séparation géographique du territoire; militarisation du Nord avec le maintien de la présence de l'armée turque; imbrication de Chypre dans le conflit gréco-turc; maintien de nationalismes exclusifs de part et d'autre; blocage politique des négociations «au sommet» entre des dirigeants qui refusent le plus souvent les compromis et gouvernent d'abord dans l'objectif de séduire leur propre communauté et d'obtenir des victoires électorales à court terme; gestion encore douloureuse des mémoires individuelles et collectives; déséquilibres économiques entre le Nord et le Sud; faiblesse des échanges au-delà de rapports convenus entre des communautés qui vivent désormais «chacune chez soi» depuis plusieurs décennies; difficultés à surmonter les idées reçues et les mythes véhiculés par les propagandes nationalistes, les télévisions, les journaux et parfois même l'éducation, etc. Mais Chypre ne se résume pas au «conflit», pas plus que le conflit ne se résume à un affrontement entre deux communautés « prises dans leur ensemble », comme le rappelle bien Gilles Bertrand en critiquant cette vision erronée.

Au-delà de l'image d'un «conflit gelé», de nombreuses évolutions éminemment positives ont eu lieu au cours des dernières décennies : ouverture de la Ligne verte en 2003 ; développement sans précédent du dialogue bicommunautaire malgré l'échec du plan Annan en 2004; consolidation de liens d'amitié de part et d'autre de la frontière et au sein de la diaspora transnationale; démocratisation progressive des institutions politiques du Nord de Chypre; recentrage des mobilisations sociales sur des enjeux socioéconomiques comme en 2001 au Nord et en 2013 au Sud; renforcement de l'internationalisation de Chypre avec l'essor de nouvelles migrations qui remettent en question les frontières communautaires 
10 Politique et sociétés à Chypre aujourd'hui

et identitaires traditionnelles; persistance d'un esprit et d'une culture pacifiste partagés. Pourtant, malgré l'ampleur des questionnements qui émergent, à partir du cas chypriote, sur la mémoire, le vivre ensemble, les aspects identitaires ou encore l'État, la politique et les sociétés de Chypre demeurent assez méconnus, notamment dans leurs aspects les plus contemporains. L'ambition de ce numéro spécial n'est pas d'apporter une étude exhaustive de la question, mais de proposer six articles qui posent un regard nouveau sur les enjeux de l'étatisation et de la militarisation du territoire à Chypre du Nord, sur la cause de la disparition, les dynamiques du rapprochement bicommunautaire, le rôle de l'ONU, les mutations de la diaspora ou encore les nouveaux enjeux énergétiques et géopolitiques liés à la découverte de gisements d'hydrocarbures au large de l'île.

\section{Étatisation et militarisation de l'espace à Chypre du Nord}

Dotée d'un gouvernement, d'une administration civile, d'un président et d'un Parlement élus de façon régulière depuis la création de l'«État turc fédéré de Chypre» en 1975, la «République turque de Chypre du Nord » (RTCN, ou Kuzey Kibrıs Türk Cumburiyet, KKTC en turc) a longtemps été dominée par Rauf Denktaş (1975-2005) et son parti de l'unité nationale (Ulusal Birlik Partisi, UBP), fidèle au nationalisme turc. De 2005 à 2009 et à la suite des élections législatives du 28 juillet 2013, l’opposition de centre gauche, menée par Özkan Yorgancioğlü du parti républicain turc (Cumburiyetci Türk Partisi, CTP), gouverna en coalition avec le parti démocrate (Demokrat Parti, DP), nationaliste, mais moins intransigeant que l'UBP, dont le dirigeant, Derviş Eroğlu, a dominé la présidence de 2010 à 2015, avant d'être battu par le candidat de gauche, Mustafa Akınc1, lors de la présidentielle d'avril 2015. Reconnue uniquement par la Turquie, la «RTCN $»$ constitue néanmoins un «État de facto », un territoire dépourvu de reconnaissance internationale, mais qui met en avant une forme de «souveraineté » interne, quoique contestée, en s'appuyant depuis la partition de 1974 sur le soutien indéfectible d'environ 30000 militaires turcs, accusés par leurs opposants d'être une force « d'occupation ».

À partir d'une perspective ethnographique, Mathieu Petithomme propose ici un article sur les dynamiques d'attachement des Chypriotes turcs à l'égard de leur administration, dans le contexte d'un État de facto non reconnu et dépendant de la Turquie depuis 1974. En s'appuyant sur des enquêtes de terrain menées entre 2012 et 2014, il s'intéresse aux affects générés par la « RTCN » sur ses agents publics et ses citoyens. Il montre que l'isolement diplomatique et économique renforce leur dépendance et leur loyauté à l'égard de leur administration, mais génère aussi malaise et insatisfaction chronique chez des citoyens conscients des limites de la 
« souveraineté » autoproclamée de leur entité politique. En effet, la bureaucratie nord-chypriote engendre une forme de schizophrénie chez ses agents: la RTCN «n'a pas d'existence légale », mais les nationalistes ont malgré tout créé une administration dotée de structures propres, avec ses logiques internes. Pour le dire simplement, «la situation d'isolement diplomatique et économique de la RTCN renforce une situation de dépendance des Chypriotes turcs vis-à-vis de leur administration qui peut déboucher sur des relations clientélistes ». Les attitudes des Chypriotes turcs envers leur administration non reconnue s'insèrent dans le cadre d'une double dépendance: ils sont dépendants vis-à-vis de leur administration dans un contexte d'isolement international et d'accès limité à des ressources, et leur administration est elle-même dépendante de la Turquie. Mathieu Petithomme montre ainsi comment les Chypriotes turcs cherchent à exploiter leurs réseaux sociaux (torpil) afin d'entrer dans l'«administration d'État », qui symbolise pour eux la promesse d'une stabilité salariale et sociale. Ils désirent intégrer la bureaucratie et développent donc largement des attitudes de révérence et de loyauté, mais désapprouvent également « l'oisiveté de leurs agents publics et $[. .$.$] nombreux sont ceux qui considèrent leur État comme inefficace et$ trop dépendant de la Turquie pour être réellement fonctionnel. » Ainsi, d'une certaine manière, « même si les documents et les procédures administratives de la RTCN ne sont pas reconnus internationalement, ils créent du sens au quotidien pour les Chypriotes turcs qui demeurent très attachés à un secteur public et à un État qu'ils critiquent pourtant allégrement. »

Dans l'article «Militarisation de l'espace et représentations sociales du conflit à Chypre du Nord », en se fondant sur des enquêtes de terrain menées à Nicosie-Nord (Lefkoşa/Lefkosia) et Famagouste (Gazimağusa/Ammochostos), il propose une analyse complémentaire à partir de l'étude de la militarisation de l'espace et de son impact psychosocial. Il montre que le contrôle territorial imposé par l'armée turque depuis 1974, même s'il demeure «illégal » en droit international et reste considéré comme une «occupation» (isgal) par les Chypriotes grecs et les militants de la paix, n'en demeure pas moins très réel et durable sur le terrain. La militarisation de l'espace participe au maintien de la domination de l'armée turque sur ce territoire contesté. En construisant des casernes et des postes militaires, en «turquifiant » les toponymes des localités, en interdisant l'accès à certaines zones rurales converties en camps d'entraînement et en affirmant son contrôle, l'armée turque instaure durablement sur le terrain sa puissance souveraine pourtant non reconnue. La militarisation de l'espace joue ainsi un rôle concret, mais aussi performatif: elle « fait exister » la « République turque de Chypre du Nord », en excluant les autorités, les institutions et les lois de la République de Chypre de toute interférence sur les procédures internes 
12 Politique et sociétés à Chypre aujourd'hui

de l'entité et les pratiques sociales des Chypriotes turcs. Elle modifie donc directement les perspectives de sortie du conflit, en rendant plus improbable le retour au statu quo ante bellum, pourtant désiré par les Chypriotes grecs et plaidé par la communauté internationale depuis des années.

Mathieu Petithomme montre également que la militarisation produit des effets politiques et sociaux durables, en contribuant au maintien de représentations associées au conflit, au désir de protection et à des sentiments d'isolement et de malaise. Le quadrillage du territoire et l'omniprésence du contrôle policier engendrent des sentiments partagés chez de nombreux Chypriotes turcs entre un «affect de remerciement» à l'égard de l'armée turque, mais aussi une distance critique envers elle. L'omniprésence dans l'espace social d'environ 30000 militaires, épaulés par la force de sécurité et les policiers chypriotes turcs sous tutelle de l'État-major, renforce le sentiment d'être surveillé en permanence.

Enfin, à travers l'ethnographie des ruines de guerre de Varosha (Maraş), on souligne dans quelle mesure la militarisation modifie les propriétés matérielles de l'espace, mais aussi les représentations que se font les Chypriotes turcs de leur propre territoire et du conflit. Ancien quartier et station balnéaire de Famagouste peuplée avant 1974 par 37000 Chypriotes grecs dont 6000 à Varosha, cette «ville fantôme » en ruines, dont l'accès est interdit par l'armée turque, est désormais une zone tampon abandonnée, susceptible de constituer un territoire clé lors d'un éventuel échange territorial qui « témoigne et traduit physiquement ce que la partition (taksim) veut dire ». Cette situation concrète produit des affects durables et ambivalents sur les perceptions des Chypriotes turcs : sentiment d'insécurité et désir de protection, malaise et perception de confinement, rejet de la militarisation, etc.

En conclusion, l'article montre les limites de la définition classique de la souveraineté fondée sur la reconnaissance internationale et refusée à la « RTCN » sur cette base. « Même s'ils sont pleinement conscients des limites de la "souveraineté" de leur entité autoproclamée, [les Chypriotes turcs] contribuent quotidiennement par leurs pratiques sociales à "vivre avec" les contraintes de la militarisation, et à "faire exister" leur "État" qui administre leur territoire, leur donne accès à l'éducation et leur fournit les services sociaux nécessaires, même s'il demeure très dépendant de la Turquie, tant financièrement, militairement que politiquement. » La souveraineté, et particulièrement sa dimension interne, « ne se fonde donc pas seulement sur un processus culturel et symbolique de reconstruction d'un imaginaire national, mais émerge aussi à travers un processus “réaliste” d'appropriation militaire, architecturale et géographique de l'espace. » 


\section{La cause de la disparition}

En s'intéressant au traitement de la cause des disparus au sein de la communauté chypriote turque, Théotime Chabre montre comment le rythme de la politique communautaire locale et des négociations sur la réunification de l'île influence l'investissement de cette cause qui concerne 1510 Chypriotes grecs et 492 Chypriotes turcs disparus entre 1963 et 1974. Il rappelle que cette question a longtemps été taboue dans la société chypriote turque, évincée de la mémoire collective et des débats publics après 1974, car trop directement liée aux souffrances individuelles du conflit. Un comité représentatif des deux communautés, le Comité pour les personnes disparues à Chypre (CMP), existe depuis 1991 dans le but d'identifier et d'inhumer les corps des disparus. Mais si la localisation des disparus a été accaparée par le discours officiel des autorités chypriotes grecques qui en ont fait une cause nationale, il faut attendre la période ouverte par l'échec du référendum de 2004 pour constater un tournant dans la saisie de cette problématique à Chypre du Nord: des associations font alors $\mathrm{du}$ sujet une cause, les administrations publiques s'impliquent et coopèrent avec le CMP et les médias locaux relayent ce changement de paradigme dans la production de la cause victimaire. En s'appuyant notamment sur un riche travail de terrain mené en 2016, sur les groupes qui participent à la constitution de la cause, Théotime Chabre interroge en fait de manière dynamique la production et la transformation des cadres mémoriels.

Il revient d'abord sur le contexte historique, à partir des affrontements de Gönyeli en 1958, et sur les deux périodes les plus intenses de la guerre civile entre les milices de l'EOKA, Ethniki Organosis Kyprion Agoniston (Organisation nationale des combattants chypriotes) et de la TMT, Türk Mukavemet Teşkilatı (Organisation turque de résistance), durant lesquelles se concentrent l'essentiel des disparus. Les Chypriotes turcs furent particulièrement touchés par les enlèvements massifs entre le 21 décembre 1963 et juin 1964 et lors de la retraite des soldats grecs entre le 14 et 15 août 1974. Les disparitions de Chypriotes grecs se concentrent surtout lors des combats de juillet 1974 et de l'intervention turque le 20 juillet après le coup d'État des partisans de l'EOKA-B contre Makarios le 15 juillet 1974. À partir de là, des récits mémoriels concurrents se sont développés au sein des deux communautés, séparées géographiquement et structurées autour d'administrations distinctes, développant des mémoires tronquées et concentrées sur leurs propres victimes.

Le récit mémoriel chypriote grec « évacue les violences de 1963-1964 pour se concentrer sur celles liées à l'invasion turque », catastrophe nationale fondatrice de la nation chypriote grecque moderne: «les Agnooumeni ("disparus") sont 
14 Politique et sociétés à Chypre aujourd'hui

sanctifiés religieusement et reconnus politiquement comme un symbole de la permanence de l'oppression turque et du martyr de Chypre ». Ils prennent une place centrale dans le récit mémoriel et les discours nationalistes et publics. À l'inverse, les autorités chypriotes turques considèrent que les disparus sont morts et que le dossier est soldé dès 1974, proposant un cadre mémoriel des violences beaucoup plus large (de 1955 à 1974) inséré dans le projet visant à motiver le départ des Chypriotes turcs pour faciliter la mise en place de l'enosis. La propagande officielle au Nord insiste sur ces massacres pour légitimer l'intervention turque: sans elle, la communauté aurait disparu; la partition de l'île serait donc une victoire inespérée permise grâce à cette puissance «bienfaitrice » et aux sacrifices des combattants (mücahit) dont les morts sont promus au rang de martyrs. Les disparus, «présentés comme des victimes, n’ont pas leur place dans ce discours victorieux » et sont donc tenus à l'écart de la propagande officielle.

Théotime Chabre rappelle comment cette mise de côté des proches de disparus sera renforcée par le système de redistribution des ressources matérielles (les propriétés abandonnées par les Chypriotes grecs) et symboliques (la reconnaissance officielle) issues de l'invasion turque: la loi 7/1974 privilégia l'aide aux familles des martyrs et des invalides, qui bénéficieront de facilités pour obtenir des emplois publics et des bourses d'études, et dans une moindre mesure, aux « victimes des événements » (essentiellement les civils), une catégorie secondaire au sein de laquelle seront reléguées les familles des disparus, ces «victimes douteuses », sur la base d'un soupçon vis-à-vis de leurs mérites de guerre.

Théotime Chabre explique aussi les raisons multiples de l'absence de mobilisation des proches de disparus jusqu'en 2002: l'État chypriote turc «s'il peut être tenu responsable du traitement réservé aux proches des victimes, n'est pas tenu directement responsable des disparitions »; la publicisation d'une cause victimaire demande «des interfaces de communication et des manifestations publiques inexistantes à Chypre-Nord dans les années 1980-1990» dans un contexte autoritaire; l'impossibilité pour les proches des victimes de saisir la justice dont relèvent les présumés coupables; le blocus et la séparation physique empêchant l'émergence d'une cause commune; la «sous-traitance de la question aux organisations internationales »; et enfin, les faibles ressources économiques des familles des disparus, principalement des femmes seules avec enfants, ayant vécu des déplacements et l'exil.

Certes, dans le cadre des négociations bicommunautaires, le Comité pour les personnes disparues de Chypre (CMP) mis en place dès 1981 associe des représentants publics des deux communautés, mais son travail s'est résumé en quelque sorte à une «coquille vide » jusqu'en 1997, en raison de blocages des 
deux côtés, puisque «aucun des deux gouvernements ne souhaitaient faire apparaître publiquement les crimes commis par des membres de sa communauté. » Mais la crise économique qui frappe le Nord de Chypre via la Turquie à la fin des années 1990, la contestation du régime de Denktaş, l'ouverture unilatérale du mur et l'alternance politique historique de 2003 entraînent une évolution du traitement de la disparition.

Dans un contexte de crise politique, la demande de l'Association des familles des martyrs, en 2002, d'égaliser les rétributions financières et morales des «victimes des événements » avec celles des martyrs marqua à la fois un tournant et une volonté du régime de « réactualiser sa légitimité » en tant que protecteur de la communauté chypriote turque. Les autorités de Chypre du Nord vont ainsi réinvestir le CMP non pas pour résoudre le crime qu'ont subi les disparus, mais pour venir en aide aux proches des disparus. L'auteur montre comment un ensemble d'acteurs vont se saisir des brèches et des possibilités ouvertes par ce nouveau contexte. Il étudie la création du groupe "Together We Can!” à partir de 2006, né de la rencontre entre «société civile», proches des disparus et militants bicommunautaires. Il évoque enfin la création d'associations de défense du souvenir des martyrs et un processus de collectivisation relative de la cause. Il propose ainsi un tour d'horizon détaillé de l'ensemble des enjeux sociaux et politiques associés à la question de la disparition à Chypre.

\section{Réussites et difficultés du rapprochement bicommunautaire}

Alexandre Lapierre s'intéresse, lui, au rôle des mouvements associatifs et des ONG en faveur du rapprochement bicommunautaire. Il montre comment la succession de négociations «au sommet» depuis 1974 entre les représentants des deux communautés n'est pas parvenue à apporter une solution. L'efficacité de la méthode proposée par les Nations unies, la force de l'UNFICYP dont le mandat est reconduit depuis 1964, et les puissances «garantes» est clairement un échec historique.

L'auteur étudie d'abord l'émergence du chypriotisme au sein de la communauté chypriote grecque dans le sillage de l'AKEL et de l'Association pour la Nouvelle Chypre, qui chercha à faire émerger une citoyenneté territoriale commune au-delà des clivages ethniques, religieux et linguistiques. Mais la partition de 1974 rendit de fait inexistants les contacts avec la communauté chypriote turque. Le chypriotisme imposa néanmoins un mouvement culturel et politique qui parvint à « sensibiliser la communauté grecque aux souffrances de l'autre communauté et à établir une distinction entre les Chypriotes turcs et les Turcs », tout en imposant les symboles de la nation chypriote et les bases du dialogue communautaire. 
Toutefois, s'il « reflète chez les Chypriotes grecs un désir de sauver l'unité de l'île par le rapprochement entre les peuples, chez les Chypriotes turcs, il traduit plutôt une différenciation en réaction à l'assimilation turque » qui leur fut imposée par les Turcs après 1974.

Ce qui apparaît de façon frappante à travers ce travail, c'est le foisonnement d'initiatives de rapprochement enclenchées par les sociétés civiles, que ce soit par les syndicats, les ONG, les associations de femmes ou encore les groupes de professeurs. Il en résulte clairement que le rapprochement bicommunautaire s'est d'abord construit «par le bas » face à des négociations au sommet et des scènes politiques bloquées. Les propositions de 1995 du All Cyprus Trade Unions Forum (un seul droit du travail, système d'assurances sociales et de salaires, liberté de mouvement, etc.), qui regroupait la plupart des syndicats de l'île, illustrèrent à elles seules l'ouverture de certains acteurs sociaux au rapprochement, malgré les restrictions politiques et les entraves à l'action de l'intersyndicale. Les enseignants, instituteurs et professeurs d'histoire ont de même été très actifs, contribuant à travers une myriade d'associations et d'initiatives, à formuler d'autres visions plus inclusives de l'histoire et de la mémoire nationale, même s'ils se sont heurtés à des instrumentalisations politiques de l'histoire. Les actions de l'association pour le dialogue historique, de l'ONG Friends of Cyprus ou encore du Cyprus Conflict Resolution Trainers Group, ont ainsi formé plusieurs générations de militants en faveur du rapprochement.

Alexandre Lapierre insiste de même sur le rôle des collectifs et des mobilisations des femmes (Association des réfugiées, Women Walk Home, Bicommunal Women's Group, etc.) dans la promotion d'une culture du compromis. Ces militantes, appuyées par des intellectuelles, ont réussi à occuper les espaces publics et à diffuser des messages de paix. Plus globalement, il est possible de dire qu'un réseau de 4000 à 5000 «militants de la paix » s'est consolidé à Chypre au cours des années 2000, principalement autour de Nicosia/Lefkoşa et d'acteurs de la société civile entretenant des relations amicales, associatives, militantes et/ou professionnelles de part et d'autre de la Ligne verte.

Les années 2003-2004 ont marqué un tournant dans le dialogue par le rétablissement de la circulation de part et d'autre de la Ligne verte en avril 2003. Malgré l'échec du référendum, le plan Annan a généré d'intenses mobilisations des opinions publiques de part et d'autre. Mais force est de constater que, après plus de quarante années de négociations infructueuses et « des dizaines d'initiatives de citoyens cherchant le rapprochement », la perspective d'une «solution » globale semble toujours autant improbable. Les activistes en faveur du rapprochement «ne représentent pas la majorité de la population, mais constituent des "minorités agissantes" », qui ont certes fait évoluer les consciences et les débats publics au Sud 
comme au Nord, mais qui se heurtent à de fortes entraves politiques, au poids des nationalismes et des conservatismes, à des sphères médiatiques et à des références culturelles relativement cloisonnées. La partition a localisé la vie quotidienne des Chypriotes grecs et turcs de 1974 à 2003, de telle sorte que le « désir de vivre ensemble n'est pas encore l'objectif de tous », contrairement à la préférence pour le statu quo et au « vivre à côté » des uns et des autres, qui semblent beaucoup plus partagés.

\section{Le conflit chypriote relocalisé}

Barbara Karatsioli étudie, quant à elle, comment le conflit chypriote se délocalise et se transforme à Londres, comment la frontière recomposée dans l'espace diasporique agit sur la frontière physique à Chypre. Elle montre d'abord comment dans l'espace diasporique chypriote à Londres, majoritairement chypriote grec, «la frontière entre Chypriotes grecs et Chypriotes turcs ne devient pertinente qu'après 1974 », produit de la guerre et de la division à Chypre; la diaspora se transforme et se réinvente «par le conflit, et ce, sans l'apport d'une migration massive de réfugiés ». Elle propose ensuite de suivre les engagements politiques transnationaux de trois Chypriotes de la diaspora dans le conflit - et la paix afin de cerner les transformations qui se font jour au sein même de la diaspora chypriote. Elle montre bien comment le sentiment national chypriote en diaspora peut être très variable suivant la destination d'installation (dans un pays du Commonwealth britannique, au Royaume-Uni ou ailleurs), ou suivant « que l'on coexiste ou pas avec l'ennemi ». Dans le cas précis de Londres, la situation de la diaspora marquée par un certain vivre ensemble «qui s'inscrit en continuité avec le passé de pré-division de Chypre » a été « en radicale opposition avec la situation présente de l'île ». L'étude de la délocalisation et de la transformation du conflit chypriote à Londres permet de comprendre «comment les migrants s'engagent dans des actions politiques transnationales pour agir sur la frontière et sur le conflit à Chypre ».

La présence chypriote croissante à Londres remonte aux années 1920-1930. Les premiers membres de la communauté diasporique furent globalement issus de mobilités liées à la pénurie de travail à Chypre. Cette diaspora se distinguait par sa proximité avec les luttes anti-impérialistes, anticoloniales et avec la lutte des classes, notamment à travers l'adhésion au parti communiste. Mais en 1974, elle s'est radicalement transformée. Barbara Karatsioli rappelle que «ce n'est pas une arrivée massive de réfugiés chypriotes grecs à Londres qui a transformé la diaspora [...] C'est le fait de devenir réfugiés in absentia qui a été décisif. Et c'est le déplacement de leur communauté, pas le leur, qui a donné le droit au nom 
18 Politique et sociétés à Chypre aujourd'hui

de "réfugiés". » Autrement dit, « de "simple" diaspora économique, la diaspora chypriote s'est réinventée en 1974 en "diaspora générée par le conflit”. » Il s'opère alors une « réorganisation interne accompagnée de bouleversements politiques [...] avec la rapide ascension de la droite nationaliste chypriote grecque... » Ceci implique notamment que la lutte des classes «subit la même "défaite" que la gauche et devient secondaire aux questions de guerre, déracinement et survie. »

Le contexte postérieur aux manifestations massives des Chypriotes turcs en 2002, puis à l'échec du plan Annan en 2004, a ouvert une nouvelle période. Dans ce cadre changé, Barbara Karatsioli interroge les manières avec lesquelles trois acteurs transnationaux non institutionnels de la diaspora agissent dans leurs actions sur les frontières. En étudiant les trajectoires de Maria, Mustafa et Safiye, elle montre que « la volonté d'agir sur le conflit à Chypre ne s'est pas seulement exprimée et élaborée en motion par le biais de la voie institutionnelle », puisque chacun à sa manière peut jouer un rôle. Loin d'en faire des idéaux types, elle montre la complexité et la diversité des trajectoires individuelles et collectives des membres de la diaspora, influencées par de multiples déterminants. Elle illustre aussi l'émergence d'une fracture importante « entre ceux qui sont devenus réfugiés par un déplacement à l'intérieur de Chypre et ceux devenus réfugiés par un déplacement à l'étranger. » Elle propose donc d'aller « au-delà d'une approche qui voit la diaspora ou les migrants comme témoins de l'histoire, officielle ou subalterne » ou d'une approche qui «place les réfugiés dans la perpétuelle recherche de l'appartenance à la maison (home) et de la patrie (homeland). » Au contraire, elle invite à s'intéresser aux actions individuelles et/ou collectives des migrants sur l'état du conflit et de la frontière, tout en situant la diaspora dans un espace transnational.

\section{Chypre, un laboratoire d'expérimentation pour l'ONU}

Gilles Bertrand interroge, lui, le rôle de l'ONU à Chypre, qui y a appliqué depuis 1964 des processus de négociation déjà expérimentés ailleurs. Chypre constitue ainsi «un laboratoire d'expérimentation de différentes approches et solutions en matière de résolution de conflit », et ce, «d'autant plus facilement qu'il s'agit d'un des rares conflits vraiment "gelés" depuis 1974 ». Les « faiseurs de paix » n'ont donc pas été sous la pression de l'urgence. Une telle situation a été une opportunité pour l'ONU, mais peut-on dire la même chose pour Chypre?

Dans une approche historique, il revient d'abord sur les perceptions erronées de la nature du conflit chypriote. Il rappelle que le conflit est le plus ancien d'Europe et que les secrétaires généraux de l'ONU successifs ont lancé 19 des 41

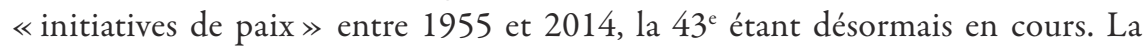


multiplicité des acteurs locaux (les deux gouvernements chypriotes grec et turc, les partis, les médias et les opinions publiques) et internationaux (l'ONU, le Royaume-Uni, la Grèce et la Turquie - les trois puissances «garantes » de l'indépendance -, les États-Unis, voire la Russie et l'Union européenne) impliqués d'une manière ou d'une autre dans la résolution du conflit a éminemment compliqué la recherche d'entente. Dès les années 1950, plusieurs lectures simplificatrices de la question chypriote s'opposent : celle du gouvernement grec et des représentants chypriotes grecs qui invoquent le droit à l'autodétermination et ne mentionnent qu'au passage la minorité «turque »; celle des Britanniques qui «mènent une politique de Divide and Rule à Chypre, en s'appuyant sur cette minorité, délibérément et par simple reproduction du modèle ottoman », même si, devant l'ONU, ils préfèrent mettre en avant la question de la stabilité au Moyen-Orient.

Ce discours est surtout entendu par le gouvernement américain qui diffuse la vision erronée suivant laquelle Chypre ne serait qu' « une pièce sur le vaste échiquier de la guerre froide ». Le simple fait que l'AKEL obtienne de bons résultats à Chypre suffit pour agiter la menace communiste. Cette vision totalement «stato-centrée » du conflit a engendré une «prophétie auto-réalisatrice » où l'ancien colonisateur donne une influence considérable aux puissances extérieures, la Grèce et la Turquie, même s'ils sont « essentiellement mus par des considérations internes »: « au lieu du partage (taksim, le mot d'ordre des nationalistes turcs chypriotes) de l'île, ce sera le partage du pouvoir, un "Yalta" qui prend la forme d'une République bicommunautaire dont l'indépendance est "garantie" par la Grèce, la Turquie et le Royaume-Uni. » Le plan dit «de partage » (Acheson) ou encore le diagnostic posé par le Conseil de sécurité des Nations unies dans sa résolution du 4 mars 1964 reprennent cette vision purement étatiste, dans le but de sauvegarder « le flanc sud-est de l'Alliance atlantique et donc de préserver les relations gréco-turques. »

Pour autant, la guerre et la partition de 1974 entraînèrent « un changement très net de perception » puisque l'URSS demeura spectatrice et que les événements montrèrent «l'impuissance des grandes puissances », ce qui laissa la place à « une médiation plus neutre, celle de l'ONU ». Mais plus de 40 ans après, parmi les gouvernements des États membres de l'ONU, et malgré l'échec de cette approche « par le haut » qui n'a donné aucun résultat jusqu'à aujourd'hui, la vision suivant laquelle « le conflit chypriote pourrait facilement se résoudre dès l'instant où les gouvernements grec, turc et de la République de Chypre se mettraient d'accord sur un nouveau mode de partage du pouvoir », demeure pourtant dominante.

«L'illusion du conflit ethnique » constitue une autre vision erronée du conflit chypriote: la sous-évaluation par les Britanniques de la montée des nationalismes 
et une lecture «de plus en plus essentialiste du conflit » à partir du prisme d'un «affrontement entre deux communautés prises dans leur ensemble » ont été des perceptions largement tronquées aux effets néanmoins durables. Non seulement la majorité des actes de violence politique ont été intracommunautaires, mais «le conflit chypriote se caractérise avant tout par des élites politiques et des formations politico-militaires qui assènent et surtout imposent par la force (faute de majorité au sein de la population) leur discours nationaliste, voire racialiste, des rapports sociaux et des conflits politiques. » Le conflit n'est donc pas «ethnique », mais travaillé par des « idéologies identitaires » forgées par les élites qui « se nourrissent et s'alimentent réciproquement». Malgré cela, Gilles Bertrand défend l'idée que les États membres de l'ONU ont accepté «le discours identitariste comme vrai», et ce, pour plusieurs raisons: «conclure que le conflit est réellement ethnique et donc dresser deux groupes homogènes l'un contre l'autre permet d'envisager des solutions "par le haut" (top-down) qui semblent plus rapides et moins coûteuses à mettre en œuvre: accords entre dirigeants. » Au contraire, analyser le conflit comme «provoqué par des élites et un discours identitaristes » conduit à «envisager des solutions plus longues [...], peut-être plus coûteuses, mais certainement plus durables: s'appuyer sur la population et en particulier sur la "société civile"; accompagner un long processus de déradicalisation de la société et de la politique; promouvoir et consolider des institutions démocratiques, etc. » Autrement dit, « une approche plus centrée sur les processus "par le bas" (bottom-up) qui inclut aussi un très long travail d'éducation. »

Ces perceptions erronées ont conduit à l'impasse des négociations officielles sous l'égide des Nations unies depuis 1965; dans la mesure où les dirigeants respectifs représentent d'abord leur communauté, ils subissent de fortes pressions en interne qui empêchent tout accord. Le scénario du «duo dysfonctionnel, comportant au moins un dirigeant apparemment conciliant et un autre manifestement obstructionniste» s'est reproduit à plusieurs reprises. Mais même lorsqu'une «fenêtre d'opportunité » est apparue, liée à l'élection de deux dirigeants sur des programmes de réunification, les cycles de négociation ont été des échecs.

Gilles Bertrand explique bien que les raisons sont à trouver dans la nature même des systèmes politiques, puisque « chacun des deux présidents doit compter sur une majorité parlementaire qui compte des partis ou des personnalités du parti du président dits "rejectionnistes" », et que ces partis utilisent quasi systématiquement leurs pouvoirs de veto. Au final, «par "dépendance au sentier" (ou esprit routinier), sous la pression des gouvernements [...] et parce qu' il s'agit de la solution la moins coûteuse au moins financièrement, les secrétaires généraux de l'ONU successifs ont accepté la méthode du dialogue au sommet. » Or tout 
le paradoxe est que celle-ci «a prouvé son inefficacité, voire sa nocivité, et pas seulement à Chypre », contrairement à des plans plus globaux, plus solides et durables, s'appuyant notamment sur la société civile et l'éducation.

\section{La découverte d'un bassin d'hydrocarbures : bienfait ou problème pour l'île?}

Le dernier article de ce numéro spécial porte sur la découverte de réserves de gaz naturel au large du Levant et ses effets pour Chypre. Joëlle Dalègre étudie ainsi les enjeux techniques et les conséquences géopolitiques de la découverte en Méditerranée orientale de ce bassin d'hydrocarbures, «qui pourrait égaler celui de la mer du Nord », et qui a initialement «provoqué l'enthousiasme des États riverains », dans l'espoir «d'atteindre l'autonomie énergétique et de faire de lucratives exportations ». Après quelques décennies de sondages épisodiques et peu fructueux, la République de Chypre décide en 2006 une première campagne de recherches, fixe sa zone économique exclusive et lance en 2007 un premier appel d'offres. En 2015, le gisement dit de Zohr est découvert au large des eaux égyptiennes, puis en 2018, le gisement Calypso: « les ressources en gaz naturel sont donc certaines, la géologie laisse encore espérer, mais l'exploitation se mêle très vite à la question du partage entre États et du droit de la mer. »

En effet, en 1982 a été signée la Convention des Nations unies sur le droit de la mer dite de Montego Bay, qui est entrée en vigueur en 1994. Mais cette convention n'a pas été signée et reste contestée par la Turquie. Par ailleurs, la Grèce et la Turquie sont en conflit depuis 1973 sur la définition de leurs eaux territoriales. La République de Chypre a néanmoins signé un certain nombre d'accords avec ses voisins, notamment l'Égypte, le Liban et Israël. La difficulté naît donc de ses rapports avec la Turquie qui « refuse de reconnaître les limites fixées par la République de Chypre et de négocier avec elle, car elle juge que cet État n'est pas un interlocuteur valable puisqu'il ne représente de facto que les Chypriotes grecs. » Agissant en protectrice des droits des Chypriotes turcs, seul État à avoir reconnu l'existence de la République turque de Chypre Nord (RTCN) proclamée unilatéralement, «elle juge qu'aucune recherche ne peut avoir lieu avant le règlement de la "question chypriote". » Le différend gréco-turc sur la délimitation des eaux territoriales renforce encore plus les difficultés.

À ces enjeux géopolitiques, s'ajoutent des questions techniques non résolues liées aux modalités d'exportation du gaz vers l'Europe. En ce sens, «l'obstacle turc » engendre une incertitude qui pèse sur les compagnies étrangères, «qui craignent pour les investissements importants que les recherches entraînent. » Non seulement la Turquie «envoie des navires de guerre menaçants à proximité 
des lieux de forage », mais l'échec des négociations sur la réunification de Chypre durant l'été 2017, le renouveau de la tension entre la Grèce et la Turquie, et les menaces rhétoriques, portent atteinte à la viabilité des perspectives d'exploitation. Joëlle Dalègre s'intéresse ainsi à la mise en valeur des ressources et aux questions pratiques qu'elle engendre: création de gazoducs, renforcement de la position de Chypre comme centre ( $h u b$ ) mondial d'échanges, etc. Au final, elle montre que cette question reste ouverte, mais que les récentes évolutions ont tendance à remettre en question l'enthousiasme initial. On espérait alors que les ressources des hydrocarbures pourraient jouer le rôle de «facilitateurs» générant une manne financière activant un intérêt commun et pouvant être redistribuée pour résoudre les problèmes financiers que provoquerait une éventuelle réunification. Mais face au blocage politique persistant, rien n'est moins sûr.

On peut donc l'affirmer pour conclure, les articles de ce numéro spécial apportent des éclairages sur un ensemble d'enjeux qui demeurent tout à fait actuels au Nord comme au Sud de l'île. Ils entrent ainsi en écho avec les récents événements sociaux et politiques à Chypre. La dépendance de la RTCN à l'égard de la Turquie, évoquée par Mathieu Petithomme, demeure une préoccupation majeure des Chypriotes turcs à l'heure où la crise économique en Turquie a des répercussions immédiates sur le nord de l'île: l'effondrement de la livre turque (-27\% en un an), monnaie officielle de la RTCN, précipite l'économie locale dans la crise. En 2018, nombre de commerçants, comme plusieurs bouchers de Nicosie-Nord, ont mis la clé sous la porte: les prix du mouton ou du bœuf ayant quasiment doublé, les Chypriotes turcs se rendent dans le Sud pour acheter leur viande moins chère. Une partie importante d'entre eux sont payés en livres turques, mais bon nombre de paiements du quotidien en RTCN se font directement en euros, en dollars ou en livres sterling, de telle sorte que les locaux ont vu leur pouvoir d'achat fondre sous l'effet de la crise en Turquie. Comble de l'ironie, même la plupart des échanges commerciaux en provenance de la Turquie, seule porte d'entrée aérienne et maritime de la RTCN sous embargo, se font en devises étrangères, ce qui entraîne une hausse généralisée des biens de consommation. La banque centrale européenne refusant par ailleurs de dialoguer directement avec la RTCN, dont $20 \%$ du budget dépend de l'aide financière de la Turquie, les Chypriotes turcs doivent donc subir une crise sur laquelle ils n'ont pas de prise. Cette situation ubuesque renforce les clivages entre les élites locales du Nord, entre ceux qui demandent encore plus «d'intégration à la Turquie » et ceux qui veulent se rapprocher du Sud pour s'émanciper de sa tutelle.

Le nouvel échec des négociations bicommunautaires durant l'été 2017 semble valider à nouveau la thèse de Gilles Bertrand sur l'inefficacité de la méthode de négociations «au sommet» entre représentants des communautés chypriotes. 
La variable des «cycles électoraux » a encore une fois joué un rôle puisque le même dirigeant, le conservateur Nicos Anastassiades, qui refusa des compromis sur la présence de l'armée turque, les ajustements territoriaux ou les garanties de sécurité lors des pourparlers de 2017, quelques mois avant l'élection présidentielle de février 2018 en République de Chypre lors de laquelle il fut réélu, a pourtant été capable de s'entendre avec le président chypriote turc, Mustafa Akincı, pour ouvrir deux nouveaux points de passage à Deryneia (près de Famagouste) le 12 novembre 2018. Cette «mesure de confiance réciproque», dans le jargon de l'ONU, montre que malgré les difficultés et les lenteurs, la paix progresse « pas à pas », notamment grâce aux acteurs de la société civile, des femmes et des ONG, comme le montre Alexandre Lapierre. Pour autant, les évolutions sont en demi-teinte puisque des processus contradictoires ont lieu certes, on ne peut que se réjouir de l'ouverture de nouveaux points de passage, ce qui porte désormais leur total à neuf. En 2017, plus de la moitié des Chypriotes grecs ont franchi l'un de ces points de passage pour se rendre du Sud vers le Nord. Mais dans le même temps, comme le rappelle Mathieu Petithomme, le processus de «turquification 》 de la population, de l'économie, des médias et des références culturelles de la RTCN se renforce. Même si ces mesures d'ouverture de la ligne favorisent les échanges intercommunautaires, d'autres processus ont tendance à «acculturer» les nouvelles générations chypriotes turques par rapport à l'héritage commun de l'île.

Tant en RTCN qu'en République de Chypre, on note néanmoins une forme de «normalisation » des vies politiques, dans le sens où «la question chypriote » (la résolution du conflit) ne constitue désormais plus qu'un enjeu parmi d'autres dans les espaces publics: pour preuve, les élections présidentielles de 2013 et de 2018 en République de Chypre, se sont surtout jouées sur les enjeux économiques. Les électeurs ont tenu le parti communiste pour responsable de la mauvaise gestion de la crise à partir de 2011 et de la crise financière en 2013, créditant au contraire les conservateurs des bénéfices de la « récupération » depuis lors et du record du secteur touristique en 2017. Les enjeux de la vie quotidienne ont aussi dominé les législatives de janvier 2018 en RTCN et engendré un gouvernement quadripartite dirigé par Tufan Erhürman (CTP). Celui-ci a certes exclu les nationalistes du parti de l'unité nationale (UBP), mais demeure potentiellement assez instable. L'élection de Niyazi Kizılyürek au Parlement européen en mai 2019, premier Chypriote turc à siéger à Bruxelles, illustra par ailleurs l'actualité de la problématique du sort des déplacés internes et des diasporas, étudiée dans ce numéro par Barbara Karatsioli. Résidant dans la partie chypriote grecque et candidat de l'AKEL, Niyazi Kizılyürek a réussi son pari de faire campagne des deux côtés de la Ligne verte et de se faire élire grâce à des voix des deux communautés (85000 Chypriotes turcs sur 300000 habitants du Nord pouvant voter lors de 
ce scrutin) : 5604 Chypriotes turcs ont ainsi franchi la frontière pour se rendre aux urnes dans la partie chypriote grecque. Mais cette «normalisation » demeure incomplète, dans la mesure où, comme l'évoque Théotime Chabre, elle s'effectue aussi à travers la dépolitisation et l'évacuation des agendas politiques des enjeux les plus douloureux liés au conflit, comme celui du traitement des disparus.

Enfin, depuis juillet 2019, dans la lignée de l'article de Joëlle Dalègre, la poursuite des activités de forage de la Turquie au large de Chypre, qui a exacerbé les tensions en Méditerranée orientale, a traduit la volonté d'Ankara d'asseoir son influence sur les projets dans ce secteur malgré l'opposition de la plupart des membres de la communauté internationale. Par la présence de ses navires, le Yavuz et le Fatih, la Turquie a essayé d'imposer une projection de puissance pour défendre les intérêts de la RTCN dans l'exploitation de ces ressources gazières. Pour justifier ses forages, la Turquie affirme ne pas être tenue par les accords de délimitation maritime entre la République de Chypre et d'autres pays riverains, arguant qu'en l'absence de résolution du conflit chypriote, il n'y a pas de moyen objectif de trancher les limites de sa ZEE.

Malgré la paix en pratique au quotidien sur l'île, on voit bien comment l'absence de résolution politique du conflit chypriote impacte encore directement ou indirectement de nombreux enjeux, de l'économie aux circulations des populations, en passant par la géopolitique et l'énergie. 